[15] T. Ak, S. B. G. Karakoc, H. Triki, Numerical simulation for treatment of dispersive shallow water waves with RosenauKdV equation, The European Physical Journal Plus 131 (10) (2016) 356-370.

[16] M. Abbas, A. Abd. Majid, A. I. Md. Ismail, A. Rashid, The application of cubic trigonometric B-spline to the numerical solution of the hyperbolic problems, Appl. Math. Comput. 239 (2014) 74-88.

[17] M. Abbas, A. Abd. Majid, A. I. Md. Ismail, A. Rashid, Numerical method using cubic trigonometric B-Spline technique for non-classical diffusion problem, Abstr. Appl. Anal. 2014 (2014), Article ID 849682, 1-11.

[18] T. Nazir, M. Abbas, A. I. Md. Ismail, A. Abd. Majid, A. Rashid, The numerical solution of advection-diffusion problems using new cubic trigonometric B-splines approach, Applied Mathematical Modelling 40 (2016) 4586-4611.

[19] S. M. Zin, A. Abd. Majid, A. I. Md. Ismail, M. Abbas, Cubic Trigonometric B-spline Approach to Numerical Solution of Wave Equation, International Journal of Mathematical, Computational, Physical, Electrical and Computer Engineering 8 (10) (2014).

[20] M. Yaseen, M. Abbas, A. I. Md. Ismail, T. Nazir, A cubic trigonometric B-spline collocation approach for the fractional sub-diffusion equations, Applied Mathematics and Computation 293 (2017) 311-319.

[21] B. J. McCartin, Theory of exponential splines, Journal of Approximation Theory 66 (1991) 1-23.

[22] M. Sakai, R. A. Usmani, A class of simple exponential B-splines and their application to numerical solution to singular perturbation problems, Numer. Math. 55 (1989) 493-500.

[23] S. C. S. Rao, M. Kumar, Exponential b- spline collocation method for self-adjoint singularly perturbed boundary value problems, Appl. Numer. Math. 58 (10) (2008) 1572-81.

[24] R. D. Multire, Solution exponential b- splines and singularly perturbed boundary problem, Numer. Algorithms 47 (2008) $191-210$.

[25] R. Mohammadi, Exponential b-spline solution of convection-diffusion equations, Appl. Math. 4 (2013) 933-44.

[26] I. Dag, O. Ersoy, Exponential cubic b-spline algorithm for equal width equation, Advanced Studies in Contemporary Mathematics 25(4) (2015) 525-35.

[27] O. Ersoy, I. Dag, The exponential cubic b-spline algorithm for Korteweg-de vries equation, Advances in Numerical Analysis 2015 (2015), Article ID 367056, 1-8.

[28] I. Dag, O. Ersoy, Numerical solution of generalized Burgers-Fisher equation by exponential cubic bspline collocation method, AIP Conference Proceedings 1648 (2015) 370008.

[29] R. Mohammadi, Exponential b-spline collocation method for numerical solution of the generalized regularized long wave equation, Chin. Phys. B 24 (5) (2015) 050206.

[30] I. Dag, O. Ersoy, The exponential cubic B-spline algorithm for Fisher equation, Chaos, Solitons and Fractals 86 (2016) 101-106.

[31] O. Ersoy, I. Dag, N. Adar, The Exponential Cubic B-spline Algorithm for Burgers's Equation, arXiv:1604.04418 Xiv.org. 


\title{
NUMERICAL TREATMENT OF FIRST ORDER MATRIX DIFFERENTIAL EQUATIONS USING DIFFERENT CUBIC B-SPLINE FUNCTIONS
}

K. R. Raslan ${ }^{1}$, A. R. Hadhoud ${ }^{2}$ and M. A. Shaalan ${ }^{3}$

${ }^{1}$ Faculty of Science, Al-Azhar University, Cairo, Egypt

${ }^{2}$ Faculty of Science, Menoufia University, Shebein El-Koom, Egypt

${ }^{3}$ Higher Technological Institute, Tenth of Ramadan City, Egypt

Received 18/3/2018Ｒevised 16/3/2018Ａccepted 9/5/2018

\begin{abstract}
The aim of the present paper is to present numerical treatments for solving Sylvester and Riccati matrix differential equations of first order with polynomial, exponential and trigonometric cubic B-spline methods. Exactness and accuracy of the proposed methods are illustrated by calculating the maximum errors. The results of numerical experiments shown by these methods are convenient to be implemented and effective numerical technique for solving matrix differential equations.
\end{abstract}

Keywords: Sylvester matrix differential equations, Riccati matrix differential equations, Polynomial cubic B-spline, Exponential cubic B-spline, Trigonometric cubic B-spline, Kronecker product, and Frobenius norm.

Mathematics Subject Classification. 41A15, 65D07, 65M70, 65M12.

\section{Introduction}

Given a boundary matrix differential equation of first order

$$
\left.\begin{array}{l}
U^{\prime}(\tau)=f(\tau, U(\tau)) \\
U(a)=U_{a}, U(b)=U_{b}
\end{array}\right\}, a \leq \tau \leq b,
$$

where matrices $U_{a}, U_{b}, U(\tau) \in C^{m \times n}$ and matrix function $f:[a, b] \times C^{m \times n} \rightarrow C^{m \times n}$, different examples of problem (??) can be found in [1]. Sylvester matrix differential equations arise in many fields of science and engineering [2,3], and Riccati matrix differential equations emerge a lot throughout science, applied mathematics and engineering. In particular, they play major roles in optimal control, filtering and estimation [4-6] and in solving linear, two-point boundary value problems of ordinary differential equations [7-10]. If $Y \in C^{m \times n}$ and $X \in C^{p \times q}$, we define the Kronecker product by $Y \otimes X[11]$

$$
Y \otimes X=\left(\begin{array}{ccc}
y_{11} X & \cdots & y_{1 n} X \\
\vdots & \ddots & \vdots \\
y_{m 1} X & \cdots & y_{m n} X
\end{array}\right)
$$

The column vector operator on a matrix $Y \in C^{n \times m}$ is given by [11]:

$$
\operatorname{Vec}(Y)=\left[\begin{array}{l}
Y_{\bullet} \\
\vdots \\
Y_{\bullet}
\end{array}\right], \quad \text { where } \quad Y_{\bullet k}=\left[\begin{array}{l}
Y_{1 k} \\
\vdots \\
Y_{m k}
\end{array}\right]
$$

Also, the derivative of a matrix $U \in C^{m \times n}$ with respect to a matrix $V \in C^{p \times q}$ is defined by [11]:

$$
\frac{\partial U}{\partial V}=\left(\begin{array}{ccc}
\frac{\partial U}{\partial v_{11}} & \cdots & \frac{\partial U}{\partial v_{1 q}} \\
\vdots & \ddots & \vdots \\
\frac{\partial U}{\partial v_{p 1}} & \cdots & \frac{\partial U}{\partial v_{p q}}
\end{array}\right), \quad \text { where } \quad \frac{\partial U}{\partial v_{n}}=\left(\begin{array}{ccc}
\frac{\partial U_{11}}{\partial v_{n}} & \cdots & \frac{\partial U_{1 n}}{\partial v_{n}} \\
\vdots & \ddots & \vdots \\
\frac{\partial U_{m 1}}{\partial v_{n}} & \cdots & \frac{\partial U_{m n}}{\partial v_{n}}
\end{array}\right)
$$

The derivative of a matrix product $V \in C^{p \times q}$ and $U \in C^{q \times v}$ with respect to another matrix $W \in C^{m \times n}$ is given by [11]:

$$
\frac{\partial V U}{\partial W}=\frac{\partial V}{\partial W}\left[I_{n} \otimes U\right]+\left[I_{m} \otimes V\right] \frac{\partial U}{\partial W},
$$


where the identity matrices of dimensions $m$ and $n$ denoted by $I_{m}$ and $I_{n}$ respectively.

The chain rule and derivative of a Kronecker product of matrices $V \otimes U$ with respect to a matrix $W$ are given by [11]:

$$
\begin{gathered}
\frac{\partial W}{\partial V}=\left[\frac{\partial[V e c(U)]^{T}}{\partial V} \otimes I_{m}\right]+\left[I_{q} \otimes \frac{\partial W}{\partial[V e c(U)]}\right], \\
\frac{\partial(V \otimes U)}{\partial W}=\frac{\partial V}{\partial W} \otimes U+\left[I_{m} \otimes U_{1}\right]\left[\frac{\partial U}{\partial W} \otimes V\right]\left[I_{n} \otimes U_{2}\right],
\end{gathered}
$$

where $V \in C^{p \times q}, U \in C^{u \times v}, W \in C^{m \times n}$ and $U_{1}, U_{2}$ are permutation matrices.

The frobenius norm of $U \in C^{m \times n}$ is given by [12]:

$$
\|U\|_{F}=\sqrt{\sum_{i=1}^{m} \sum_{j=1}^{n}\left|u_{i j}\right|^{2}} .
$$

The Frobenius norm and 2-norm holds [12]:

$$
\|U\|_{2} \leq\|U\|_{F} \leq \sqrt{n}\|U\|_{2}
$$

Cubic splines are discussed in [13-15], matrix differential equations are studied in [16-19] and exponential cubic B-splines are piecewise polynomial functions containing a free parameter and its properties are presented in [20]. The exponential and trigonometric cubic B-spline methods are studied to solve numerical solutions of various ordinary and partial differential equations [21-27] and the sextic and septic B-spline methods are introduced to solve Rosenau-KdV equation [28, 29]. This paper is organized as follows: In section 2, we present the polynomial, exponential and trigonometric cubic B-spline methods. In section 3, some numerical examples are discussed. Finally, the conclusion of this study is given in section 4 .

\section{Description of cubic B-spline methods}

Firstly, we assume that the problem domain $[a, b]$ is equally divided into $N$ subintervals $\left[\tau_{i}, \tau_{i+1}\right], i=0,1, \ldots, N-1$ by the knots $\tau_{i}=a+i h$ where $a=\tau_{0}<\tau_{1}<\cdots<\tau_{N-1}<\tau_{N}=b$ and the step size $h=\frac{b-a}{N}$. Then cubic B-spline collocation methods for solving matrix boundary value problems (1) numerically are presented.

\subsection{Polynomial cubic B-spline method (PCBSM)}

The polynomial cubic B-spline can be defined as follows:

$$
B_{i}(\tau)=\frac{1}{h^{3}} \begin{cases}\left(\tau-\tau_{i-2}\right)^{3} & \tau \in\left[\tau_{i-2}, \tau_{i-1}\right], \\ h^{3}+3 h^{2}\left(\tau-\tau_{i-1}\right)+3 h\left(\tau-\tau_{i-1}\right)^{2}-3\left(\tau-\tau_{i-1}\right)^{3} & \tau \in\left[\tau_{i-1}, \tau_{i}\right], \\ h^{3}+3 h^{2}\left(\tau_{i+1}-\tau\right)+3 h\left(\tau_{i+1}-\tau\right)^{2}-3\left(\tau_{i+1}-\tau\right)^{3} & \tau \in\left[\tau_{i}, \tau_{i+1}\right] \\ \left(\tau_{i+2}-\tau\right)^{3} & \tau \in\left[\tau_{i+1}, \tau_{i+2}\right], \\ 0 & \text { elsewhere. } \\ (i=-1,0, \cdots, N+1) . & \end{cases}
$$

We consider the spline function is interpolation to the solutions $\stackrel{k l}{u}(\tau)$ of the problem (1):

$$
{ }^{k l}(\tau)=\sum_{i=-1}^{N+1}{ }^{k l} \omega_{i}(\tau) P C B_{i}(\tau) ; 1 \leq k \leq n, 1 \leq l \leq m
$$

where constants $\stackrel{k l}{\underset{\omega}{\omega}}(\tau)$ 's are be determined. To solve boundary matrix differential equation of first order, we find $P C B_{i}$ and $P C B_{i}^{\prime}$ at the nodal points are needed. Their coefficients are summarized in Table 1. 
Table 1. values of $P C B_{i}$ and $P C B_{i}^{\prime}$.

\begin{tabular}{|c|c|c|c|c|c|}
\hline$\tau$ & $\tau_{i-2}$ & $\tau_{i-1}$ & $\tau_{i}$ & $\tau_{i+1}$ & $\tau_{i+2}$ \\
\hline$P C B_{i}$ & 0 & 1 & 4 & 1 & 0 \\
\hline$P C B_{i}^{\prime}$ & 0 & $-\frac{3}{h}$ & 0 & $\frac{3}{h}$ & 0 \\
\hline
\end{tabular}

Using Eqs. (??) and (??), the values of $u_{i}^{k l}$ and their first derivatives at the knots are

$$
\left.\begin{array}{l}
\stackrel{k l}{u_{i}}=\omega_{i-1}^{k l}+4 \stackrel{k l}{\omega_{i}}+\omega_{i+1}^{k l} \\
k l \\
u_{i}^{\prime}=-\frac{3}{h} \omega_{i-1}^{k l}+\frac{3}{h} \omega_{i+1}^{k l}
\end{array}\right\}, i=0,1, \ldots, N .
$$

Substituting from Eq. (??) in Eq. (??) we find,

$$
\left[-\frac{3}{h} \omega_{i-1}^{k l}+\frac{3}{h} \omega_{i+1}^{k l}=f\left(i h, \omega_{i-1}^{k l}+4 \stackrel{k l}{\omega} \omega_{i}+\omega_{i+1}^{k l}\right),\right]
$$

where $i=0,1, \ldots, N, k=1,2, \ldots, n$ and $l=1,2, \ldots, m$.

and the boundary conditions are given as

$$
\begin{aligned}
& \stackrel{k l}{k l} \stackrel{k l}{\omega_{-1}}+4 \omega_{0}+\stackrel{k l}{\omega_{a}}, \\
& k l \\
& \omega_{N-1}^{k l}+4 \stackrel{k l}{\omega}_{N}+\omega_{N+1}^{k l}=\stackrel{k l}{\omega_{b}} .
\end{aligned}
$$

Solving the system of Eqs. (??) in $\omega_{-1}^{k l}$ and $\omega_{N+1}^{k l}$, the linear algebraic system of Eqs. (??) can be converted to the following matrix form ;

$$
\stackrel{k l}{A} \stackrel{k l}{\omega}=\stackrel{k l}{F}, 1 \leq k \leq n, 1 \leq l \leq m .
$$

where $\stackrel{k l}{A}$ is an $(N+1) \times(N+1)$ matrix, $\stackrel{k l}{\omega}$ is an $N+1$ dimensional vector with components $\stackrel{k l}{\omega}$ and the right hand side $\stackrel{k l}{F}$ is an $N+1$ dimensional vector;

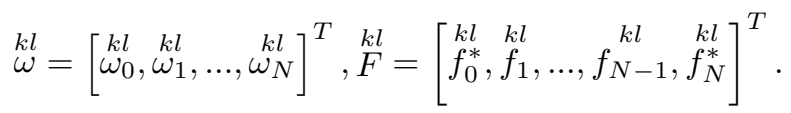

\subsection{Exponential cubic B-spline method (ECBSM)}

The exponential cubic B-spline can be defined as follows:

$$
\operatorname{ECB}_{i}(\tau)= \begin{cases}w_{1}\left[\left(\tau_{i-2}-\tau\right)-\frac{1}{\eta}\left(\sinh \left(\eta\left(\tau_{i-2}-\tau\right)\right)\right)\right] & \tau \in\left[\tau_{i-2}, \tau_{i-1}\right] \\ w_{2}+w_{3}\left(\tau_{i}-\tau\right)+w_{4} e^{\eta\left(\tau_{i}-\tau\right)}+w_{5} e^{-\eta\left(\tau_{i}-\tau\right)} & \tau \in\left[\tau_{i-1}, \tau_{i}\right] \\ w_{2}+w_{3}\left(\tau-\tau_{i}\right)+w_{4} e^{\eta\left(\tau-\tau_{i}\right)}+w_{5} e^{-\eta\left(\tau-\tau_{i}\right)} & \tau \in\left[\tau_{i}, \tau_{i+1}\right] \\ w_{1}\left[\left(\tau-\tau_{i+2}\right)-\frac{1}{\eta}\left(\sinh \left(\eta\left(\tau-\tau_{i+2}\right)\right)\right]\right. & \tau \in\left[\tau_{i+1}, \tau_{i+2}\right] \\ 0 & \text { elsewhere } \\ (i=-1,0, \cdots, N+1) . & \end{cases}
$$

where,

$$
\begin{aligned}
& w_{1}=\frac{\eta}{2(\eta h C-S)}, w_{2}=\frac{\eta h C}{\eta h C-S}, w_{3}=\frac{\eta}{2}\left[\frac{C(C-1)+S^{2}}{(\eta h C-S)(1-C)}\right] \\
& w_{4}=\frac{1}{4}\left[\frac{e^{-\eta h}(1-C)+S\left(e^{-\eta h}-1\right)}{(\eta h C-S)(1-C)}\right], w_{5}=\frac{1}{4}\left[\frac{e^{\eta h}(C-1)+S\left(e^{\eta h}-1\right)}{(\eta h C-S)(1-C)}\right], C=\operatorname{Cosh}(\eta h), S=\operatorname{Sinh}(\eta h),
\end{aligned}
$$

and $\eta$ is a free parameter.

We consider the spline function is interpolation to the solutions $\stackrel{k l}{u}(\tau)$ of the problem (??):

$$
{ }_{u}^{k l}(\tau)=\sum_{i=-1}^{N+1}{ }_{\zeta}^{k l}(\tau) E C B_{i}(\tau) ; 1 \leq k \leq n, 1 \leq l \leq m,
$$


where constants ${ }_{\zeta}^{k l}(\tau)$ 's are be determined. To solve boundary matrix differential equation of first order, we find $E C B_{i}$ and $E C B_{i}^{\prime}$ at the nodal points are needed. Their coefficients are summarized in Table 2.

Table 2. Values of $E C B_{i}$ and $E C B_{i}^{\prime}$

\begin{tabular}{|c|c|c|c|c|c}
\hline$\tau$ & $\tau_{i-2}$ & $\tau_{i-1}$ & $\tau_{i}$ & $\tau_{i+1}$ & $\tau_{i+2}$ \\
\hline$E C B_{i}$ & 0 & $\beta_{1}$ & 1 & $\beta_{1}$ & 0 \\
\hline$E C B_{i}^{\prime}$ & 0 & $-\beta_{2}$ & 0 & $\beta_{2}$ & 0 \\
\hline
\end{tabular}

where,

$$
\beta_{1}=\frac{S-\eta h}{2(\eta h C-S)}, \beta_{2}=\frac{\eta(C-1)}{2(\eta h C-S)}, \beta_{3}=\frac{\eta^{2} S}{2(\eta h C-S)}, \beta_{4}=\frac{-\eta^{2} S}{\eta h C-S} .
$$

Using Eqs. (17) and (18), the values of $u_{i}^{k l}$ and their first derivatives at the knots are

$$
\left.\begin{array}{l}
\begin{array}{l}
k l \\
u_{i}
\end{array}=\beta_{1} \zeta_{i-1}^{k l}+{ }_{k l}^{k l} \zeta_{i}+\beta_{1} \zeta_{i+1}^{k l} \\
k l \\
u_{i}^{\prime}=-\beta_{2} \zeta_{i-1}+\beta_{2} \zeta_{i+1}
\end{array}\right\}, i=0,1, \ldots, N .
$$

Substituting from Eq. (??) in Eq. (??) we find,

$$
\left[-\beta_{2} \zeta_{i-1}^{k l}+\beta_{2} \zeta_{i+1}^{k l}=f\left(i h, \beta_{1} \zeta_{i-1}^{k l} \stackrel{k l}{k l} \zeta_{i}+\beta_{1} \zeta_{i+1}^{k l}\right),\right]
$$

where $i=0,1, \ldots, N, k=1,2, \ldots, n$ and $l=1,2, \ldots, m$.

and the boundary conditions are given as

$$
\begin{gathered}
\stackrel{k l}{k l} \stackrel{k l}{k l} \stackrel{k l}{\zeta_{1}}=\stackrel{k l}{u_{a},}, \\
\beta_{1} \zeta_{-1}+\stackrel{\zeta_{0}+\beta_{1}}{\zeta_{1}} \stackrel{k l}{k l}+{ }_{k l}^{k l} \\
\beta_{1} \zeta_{N-1}+\zeta_{N}+\beta_{1} \zeta_{N+1}=u_{b} .
\end{gathered}
$$

Solving the system of Eqs. (21) in $\zeta_{-1}^{k l}$ and $\zeta_{N+1}^{k l}$, the linear algebraic system of Eqs. (??) can be converted to the following matrix form ;

$$
\stackrel{k l}{A} \stackrel{k l}{\zeta}=\stackrel{k l}{F}, 1 \leq k \leq n, 1 \leq l \leq m
$$

where $\stackrel{k l}{A}$ is an $(N+1) \times(N+1)$ matrix, $\stackrel{k l}{\zeta}$ is an $(N+1)$ dimensional vector with components $\stackrel{k l}{\zeta}$ and the right hand side $\stackrel{k l}{F}$ is an $(N+1)$ dimensional vector;

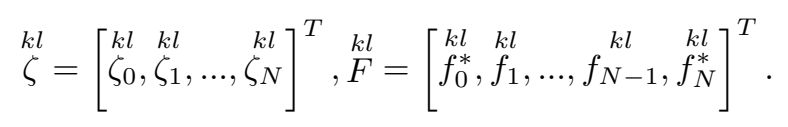

\subsection{Trigonometric cubic B-spline method (TCBSM)}

The trigonometric cubic B-spline can be defined as follows:

$$
T C B_{i}(\tau)=\frac{1}{\rho} \begin{cases}\varphi^{3}\left(\tau_{i-2}\right) & \tau \in\left[\tau_{i-2}, \tau_{i-1}\right], \\ \varphi\left(\tau_{i-2}\right)\left[\varphi\left(\tau_{i-2}\right) \vartheta\left(\tau_{i}\right)+\varphi\left(\tau_{i-1}\right) \vartheta\left(\tau_{i+1}\right)\right]+\varphi^{2}\left(\tau_{i-1}\right) \vartheta\left(\tau_{i+2}\right) & \tau \in\left[\tau_{i-1}, \tau_{i}\right], \\ \vartheta\left(\tau_{i+2}\right)\left[\vartheta\left(\tau_{i+2}\right) \varphi\left(\tau_{i}\right)+\vartheta\left(\tau_{i+1}\right) \varphi\left(\tau_{i-1}\right)\right]+\vartheta^{2}\left(\tau_{i+1}\right) \varphi\left(\tau_{i-2}\right) & \tau \in\left[\tau_{i}, \tau_{i+1}\right], \\ \vartheta^{3}\left(\tau_{i+2}\right) & \tau \in\left[\tau_{i+1}, \tau_{i+2}\right], \\ 0 & \text { elsewhere. } \\ (i=-1,0,1, \cdots, N+1) . & \end{cases}
$$

where,

$$
\rho=\sin \left(\frac{h}{2}\right) \sin (h) \sin \left(\frac{3 h}{2}\right), \varphi\left(\tau_{i}\right)=\sin \left(\frac{\tau-\tau_{i}}{2}\right), \vartheta\left(\tau_{i}\right)=\sin \left(\frac{\tau_{i}-\tau}{2}\right)
$$


we consider the spline function is interpolation to the solutions $\stackrel{k l}{k}(\tau)$ of the problem (1):

$$
{ }_{u}^{k l}(\tau)=\sum_{i=-1}^{N+1} \mathrm{~T}_{i}^{k l}(\tau) B_{i}(\tau) \quad ; 1 \leq k \leq n, 1 \leq l \leq m
$$

where constants $\mathrm{T}_{i}^{k l}(\tau)$ 's are be determined. To solve boundary matrix differential equation of first order, we find $T C B_{i}$ and $T C B_{i}^{\prime}$ at the nodal points are needed. Their coefficients are summarized in Table 3.

Table 3. values of $T C B_{i}$ and $T C B_{i}^{\prime}$.

$\mid$\begin{tabular}{c|c|c|c|c|c}
\hline$\tau$ & $\tau_{i-2}$ & $\tau_{i-1}$ & $\tau_{i}$ & $\tau_{i+1}$ & $\tau_{i+2}$ \\
\hline$T C B_{i}$ & 0 & $\Omega_{1}$ & $\Omega_{2}$ & $\Omega_{1}$ & 0 \\
\hline$T C B_{i}^{\prime}$ & 0 & $-\Omega_{3}$ & 0 & $\Omega_{3}$ & 0 \\
\hline
\end{tabular}

where,

$$
\begin{aligned}
& \Omega_{1}=\frac{\sin ^{2}\left(\frac{h}{2}\right)}{\sin (h) \sin \left(\frac{3 h}{2}\right)}, \Omega_{2}=\frac{2}{1+\cos (h)}, \Omega_{3}=\frac{3}{4 \sin \left(\frac{3 h}{2}\right)}, \Omega_{4}=\frac{3(1+3 \cos (h))}{16 \sin ^{2}\left(\frac{h}{2}\right)\left(2 \cos \left(\frac{h}{2}\right)+\cos \left(\frac{3 h}{2}\right)\right)}, \\
& \Omega_{5}=\frac{-3 \cos ^{2}\left(\frac{h}{2}\right)}{\sin ^{2}\left(\frac{h}{2}\right)(2+4 \cos (h))} .
\end{aligned}
$$

Using Eqs. (??) and (??), the values of $u_{i}$ and their first derivatives at the knots are

$$
\left.\begin{array}{l}
\stackrel{k l}{u_{i}}=\Omega_{1} \mathrm{~T}_{i-1}^{k l}+\Omega_{2} \stackrel{k l}{\mathrm{~T}_{i}+\Omega_{1}} \stackrel{k l}{k l} \mathrm{~T}_{i+1}^{k l} \\
u_{i}^{\prime}=-\Omega_{3} \mathrm{~T}_{i-1}^{k l}+\Omega_{3} \mathrm{~T}_{i+1}
\end{array}\right\}, i=0,1, \ldots, N
$$

Substituting from Eq. (??) in Eq. (??) we find,

$$
\left[-\Omega_{3} \mathrm{~T}_{i-1}^{k l}+\Omega_{3} \mathrm{~T}_{i+1}^{k l}=f\left(i h, \Omega_{1} \mathrm{~T}_{i-1}^{k l}+\Omega_{2} \mathrm{~T}_{i}^{k l}+\Omega_{1} \mathrm{~T}_{i+1}^{k l}\right),\right]
$$

where $i=0,1, \ldots, N, k=1,2, \ldots, n$ and $l=1,2, \ldots, m$.

and the boundary conditions are given as

$$
\begin{aligned}
& \stackrel{k l}{k l}+\Omega_{2} \mathrm{~T}_{0}^{k l}+\Omega_{1} \stackrel{k l}{\mathrm{~T}_{1}}=\stackrel{k l}{u_{a}}, \\
& \Omega_{1} \mathrm{~T}_{k l}^{k l}+k l \\
& \Omega_{1} \mathrm{~T}_{N-1}^{k l}+\Omega_{2} \mathrm{~T}_{N}+\Omega_{1} \mathrm{~T}_{N+1}^{k l}=u_{b} .
\end{aligned}
$$

Solving the system of Eqs. (??) in $\mathrm{T}_{-1}^{k l}$ and $\mathrm{T}_{N+1}^{k l}$, the linear algebraic system of Eqs. (??) can be converted to the following matrix form;

$$
\stackrel{k l}{A \mathrm{k}} \mathrm{T}=\stackrel{k l}{F}, 1 \leq k \leq n, 1 \leq l \leq m
$$

where $\stackrel{k l}{A}$ is an $(N+1) \times(N+1)$ matrix, $\stackrel{k l}{\mathrm{~T}}$ is an $(N+1)$ dimensional vector with components $\stackrel{k l}{\mathrm{~T}_{i}}$ and the right hand side $\stackrel{k l}{F}$ is an $(N+1)$ dimensional vector;

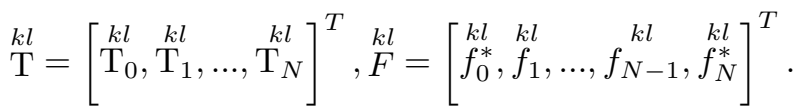

\section{$3 \quad$ Numerical examples}

In this section, we present examples of matrix differential equations of first order to explore the efficiency and accuracy of the proposed methods using Frobenius norm of the difference between approximate solution and exact solution at each point in the interval $[a, b]$ taking a suitable step size $h$ and the results are generated with Mathematica using Find Root function to solve the emerging algebraic equations. 
Example 1. We examine the linear Sylvester matrix differential equation of the type [18]

$$
U^{\prime}(\tau)=A(\tau) U(\tau)+U(\tau) B(\tau)+C(\tau) ; a \leq \tau \leq b, U(\tau), A(\tau), B(\tau), C(\tau) \in C^{n \times n},
$$

where,

$$
A(\tau)=\left(\begin{array}{cc}
0 & \tau e^{-\tau} \\
\tau & 0
\end{array}\right), B(\tau)=\left(\begin{array}{ll}
0 & \tau \\
0 & 0
\end{array}\right), C(\tau)=\left(\begin{array}{cc}
-\left(1+\tau^{2}\right) e^{-\tau} & -2 \tau e^{-\tau} \\
1-\tau e^{-\tau} & -\tau^{2}
\end{array}\right), 0 \leq \tau \leq 1
$$

This example has an exact solution $U(\tau)=\left(\begin{array}{cc}e^{-\tau} & 0 \\ \tau & 1\end{array}\right)$. Thus, we can compare our numerical estimates with this solution to obtain the exact errors of the approximation which summarized in Table 4 and figure 1.

For a free equilibrium pointsu ${ }_{j} \equiv 0,(j=1,2, \ldots, 4)$, we find that the Jacobian matrix of the Eq. (??) is

$$
\left(\begin{array}{cccc}
0 & 0 & \tau e^{-\tau} & 0 \\
\tau & 0 & 0 & \tau e^{-\tau} \\
\tau & 0 & 0 & 0 \\
0 & \tau & \tau & 0
\end{array}\right),
$$

and its eigenvalues are evaluated from the equation

$$
\lambda^{4}-2 \tau^{2} e^{-\tau} \lambda^{2}+\tau^{4} e^{-\tau}=0
$$

where $\lambda_{1,2}=-\tau e^{-\tau / 2}$ and $\lambda_{3,4}=\tau e^{-\tau / 2}$ have distinct signs for any value of $\tau$ in the interval $(0,1]$, then the equilibrium points $u_{j}=0$ of the Eq. (??) are unstable.; $(j=1,2, \ldots, 4)$.

Table 4. Comparison of maximum absolute errors for Example 1.

\begin{tabular}{|c|c|c|c|c|}
\hline$\tau$ & $\begin{array}{c}\text { Ploynomial Cubic } \\
\text { B-spline errors } \\
(\text { PCBSM) }\end{array}$ & $\begin{array}{c}\text { Exponential Cubic } \\
\text { B-spline errors } \\
(\text { ECBSM) }\end{array}$ & $\begin{array}{c}\text { Trigonometric Cubic } \\
\text { B-spline errors } \\
(\text { TCBSM })\end{array}$ & $\begin{array}{c}\text { Cubic spline } \\
\text { errors [18] } \\
(\text { CSM })\end{array}$ \\
\hline 0 & $6.28559 \times 10^{-16}$ & $1.11022 \times 10^{-16}$ & $1.11022 \times 10^{-16}$ & $1.33472 \times 10^{-6}$ \\
0.1 & $2.79999 \times 10^{-6}$ & $8.83553 \times 10^{-14}$ & $1.14486 \times 10^{-5}$ & $1.33472 \times 10^{-6}$ \\
0.2 & $7.66359 \times 10^{-8}$ & $2.38400 \times 10^{-15}$ & $3.17897 \times 10^{-7}$ & $1.24450 \times 10^{-6}$ \\
0.3 & $2.79728 \times 10^{-6}$ & $8.94896 \times 10^{-14}$ & $1.14921 \times 10^{-5}$ & $1.24450 \times 10^{-6}$ \\
0.4 & $1.00154 \times 10^{-7}$ & $3.57633 \times 10^{-15}$ & $4.19200 \times 10^{-7}$ & $1.17402 \times 10^{-6}$ \\
0.5 & $2.79649 \times 10^{-6}$ & $9.04182 \times 10^{-14}$ & $1.15252 \times 10^{-5}$ & $1.17402 \times 10^{-6}$ \\
0.6 & $8.73882 \times 10^{-8}$ & $3.31691 \times 10^{-15}$ & $3.68200 \times 10^{-7}$ & $1.12331 \times 10^{-6}$ \\
0.7 & $2.79628 \times 10^{-6}$ & $9.10099 \times 10^{-14}$ & $1.15484 \times 10^{-5}$ & $1.12331 \times 10^{-6}$ \\
0.8 & $5.09836 \times 10^{-8}$ & $2.26313 \times 10^{-15}$ & $2.15412 \times 10^{-7}$ & $1.09412 \times 10^{-6}$ \\
0.9 & $2.79623 \times 10^{-6}$ & $9.13946 \times 10^{-14}$ & $1.15641 \times 10^{-5}$ & $1.09412 \times 10^{-6}$ \\
1.0 & $3.18888 \times 10^{-16}$ & $1.11022 \times 10^{-16}$ & $5.55112 \times 10^{-17}$ & \\
\hline
\end{tabular}

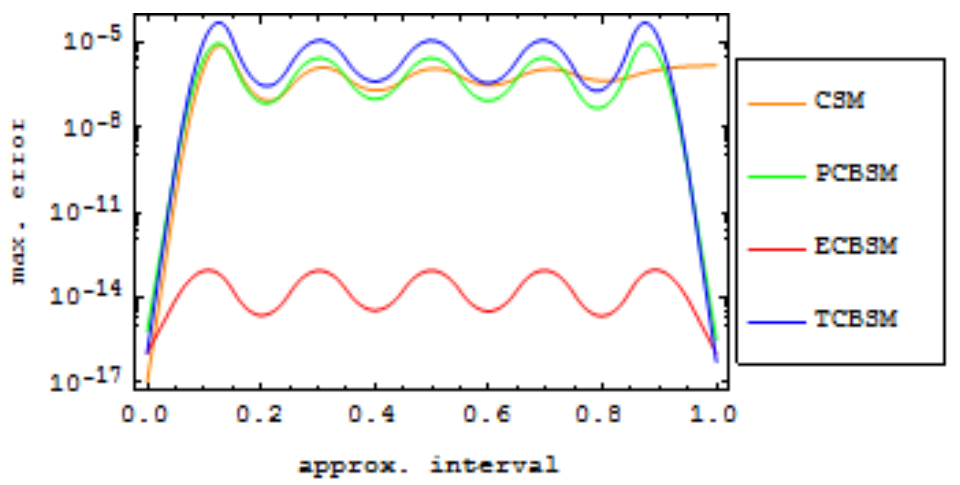

Figure 1. Comparison of maximum absolute errors for Sylvester matrix differential equation in the interval $[0,1]$ with step size $h=0.1$. 
Example 2. We investigate the rectangular non-symmetric Riccati matrix differential equation of the type [18]

$$
\begin{aligned}
& U^{\prime}(\tau)=C(\tau)-D(\tau) U(\tau)-U(\tau) A(\tau)-U(\tau) B(\tau) U(\tau) ; U(\tau) \in C^{n \times m}, U(\tau) \in C^{n \times m}, A(\tau) \in C^{m \times m}, \\
& B(\tau) \in C^{m \times n}, C(\tau) \in C^{n \times m}, D(\tau) \in C^{n \times n}, 0 \leq \tau \leq a
\end{aligned}
$$

where,

$$
\begin{aligned}
& A(\tau)=\left(\begin{array}{ll}
-\tau & 0 \\
-\tau & \tau
\end{array}\right), B(\tau)=\left(\begin{array}{cc}
-\tau^{2} & -2 \\
0 & 1
\end{array}\right), D(\tau)=\left(\begin{array}{cc}
-1 & -\tau^{-2} \\
\tau & \tau
\end{array}\right) \\
& C(\tau)=\left(\begin{array}{cc}
\tau\left(-e^{\tau}+\tau e^{\tau}-\tau^{3}\right) & \tau\left(2 e^{\tau}-\tau^{2}\right) \\
\tau(1-\tau)\left(2+\tau+2 \tau^{2}\right) & 1+\tau^{2}(3-2 \tau)+e^{\tau}\left(\tau-\tau^{4}\right)
\end{array}\right), 0 \leq \tau \leq 0.1 .
\end{aligned}
$$

This example has an exact solution $U(\tau)=\left(\begin{array}{cc}0 & e^{\tau} \\ \tau^{2} & \tau\end{array}\right)$. Thus, we can compare our numerical estimates with this solution to obtain the exact errors of the approximation which summarized in Table 5 and figure 2.

For a free equilibrium points $u_{j} \equiv 0,(j=1,2, \ldots, 4)$, we find that the Jacobian matrix of the Eq. (33) is

$$
\left(\begin{array}{cccc}
1+\tau & \tau & \tau^{2} & 0 \\
0 & 1-\tau & 0 & \tau^{2} \\
-\tau & 0 & 0 & \tau \\
0 & -\tau & 0 & -2 \tau
\end{array}\right)
$$

and its eigenvalues are evaluated from the equation

\begin{tabular}{|c|c|c|c|c|}
\hline$\tau$ & $\begin{array}{c}\text { Ploynomial Cubic } \\
\text { B-spline errors } \\
(P C B S M)\end{array}$ & $\begin{array}{c}\text { Exponential Cubic } \\
\text { B-spline errors } \\
\text { (ECBSM) }\end{array}$ & $\begin{array}{c}\text { Trigonometric Cubic } \\
\text { B-spline errors } \\
(T C B S M)\end{array}$ & $\begin{array}{c}\text { Cubic spline } \\
\text { errors }[18] \\
\text { (CSM) }\end{array}$ \\
\hline 0 & $2.1684 \times 10^{-19}$ & $4.33681 \times 10^{-19}$ & $1.11023 \times 10^{-16}$ & 0 \\
\hline 0.01 & $7.79457 \times 10^{-11}$ & $2.01112 \times 10^{-15}$ & $5.22446 \times 10^{-10}$ & $1.39903 \times 10^{-10}$ \\
\hline 0.02 & $1.42204 \times 10^{-13}$ & $7.42212 \times 10^{-19}$ & $5.83501 \times 10^{-13}$ & $2.9602 \times 10^{-12}$ \\
\hline 0.03 & $7.74756 \times 10^{-11}$ & $1.56922 \times 10^{-15}$ & $5.22841 \times 10^{-10}$ & $1.41976 \times 10^{-10}$ \\
\hline 0.04 & $2.14239 \times 10^{-13}$ & $1.14405 \times 10^{-18}$ & $8.73255 \times 10^{-13}$ & $5.91358 \times 10^{-12}$ \\
\hline 0.05 & $7.69626 \times 10^{-11}$ & $1.78941 \times 10^{-15}$ & $5.23167 \times 10^{-10}$ & $1.44084 \times 10^{-10}$ \\
\hline 0.06 & $2.14852 \times 10^{-13}$ & $1.0766 \times 10^{-18}$ & $8.71469 \times 10^{-13}$ & $8.85918 \times 10^{-12}$ \\
\hline 0.07 & $7.64061 \times 10^{-11}$ & $1.79108 \times 10^{-15}$ & $5.23433 \times 10^{-10}$ & $1.46223 \times 10^{-10}$ \\
\hline 0.08 & $1.43865 \times 10^{-13}$ & $1.39261 \times 10^{-17}$ & $5.79685 \times 10^{-13}$ & $1.17952 \times 10^{-11}$ \\
\hline 0.09 & $7.5805 \times 10^{-11}$ & $1.78943 \times 10^{-15}$ & $5.23647 \times 10^{-10}$ & $1.4839 \times 10^{-10}$ \\
\hline 0.1 & $1.73472 \times 10^{-18}$ & $1.73472 \times 10^{-18}$ & $2.22485 \times 10^{-16}$ & $1.4721 \times 10^{-11}$ \\
\hline
\end{tabular}

$$
\lambda^{4}+2(-1+\tau) \lambda^{3}+\left(1-4 \tau-\tau^{2}+2 \tau^{3}\right) \lambda^{2}+2\left(\tau-2 \tau^{3}+\tau^{4}\right) \lambda+\left(-2+2 \tau+\tau^{2}\right) \tau^{4}=0
$$

where $\lambda_{1,2}=\frac{1}{2}\left(1-3 \tau \pm \sqrt{1+2 \tau+\tau^{2}-4 \tau^{3}}\right)$ and $\lambda_{3,4}=\frac{1}{2}\left(1+\tau \pm \sqrt{1+2 \tau+\tau^{2}-4 \tau^{3}}\right)$ have distinct sign for any value of $\tau$ in the interval $(0,0.1]$, then the equilibrium points $u_{j}=0$ of the Eq. (33) are unstable.; $(j=1,2, \ldots, 4)$.

Table 5. Comparison of maximum absolute errors for Example 2.

\section{Conclusion}

In this article, we have examined scheme treat numerically with the first-order matrix differential equations by cubic Bspline method and exponential and trigonometric cubic B-spline methods. From the computational results, we can view that the cubic B-spline and exponential and trigonometric cubic B-splines are summarized and easy to apply and the errors are acceptable. The numerical experiments are compared with the analytic solutions by finding Frobenius norm and are compared with Ref. [18] as shown in Tables (3-5) and Figures (1, 2).

\section{Acknowledgments}

The authors would like to express their sincere thanks to the reviewers for their careful reading, additions valuable scientific comments and suggestions. 


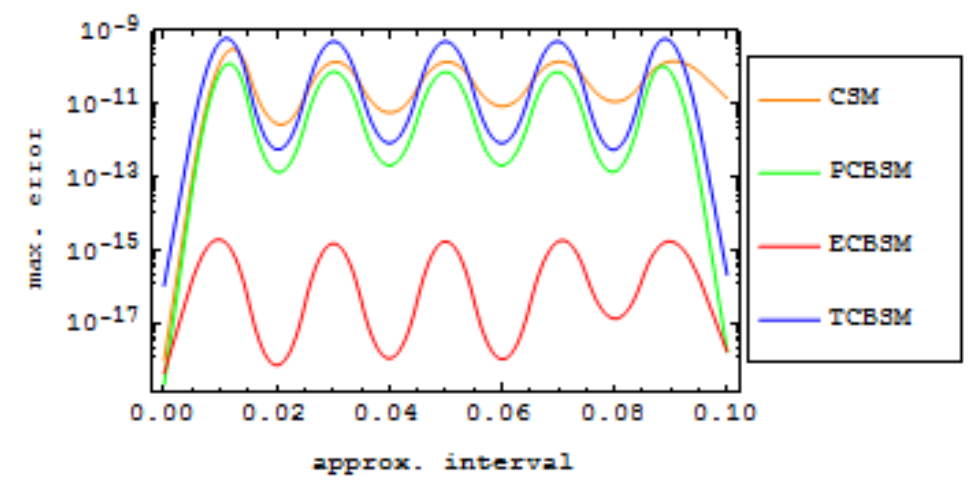

Figure 2. Comparison of maximum absolute errors for Riccati matrix differential equation in the interval $[0,1]$ with step size $h=0.01$.

\section{References}

[1] U. M. Ascher, R. M. M. Mattheij, R. D. Russell, Numerical Solutions of Boundary Value Problems for Ordinary Differential Equations, Prentice Hall, New Jersey, 1988.

[2] L. Jodar, E. Ponsoda, Computing continuous numerical solutions of matrix differential equations, Comput. Math. Appl. 29 (4) (1995) 73-84.

[3] L. V. Fausett, Sylvester matrix differential equations: analytical and numerical solutions, International Journal of Pure and Applied Mathematics, Volume 53 (1) (2009) 55-68.

[4] H. Kwakernaak, R. Sivan, Linear Optimal Control Systems, Wiley Interscience, New York, 1972.

[5] J. L. Casti, Dynamical Systems and Their Applications: Linear Theory, Academic Press, New York, 1977.

[6] L. Jodar, J. C. C. Lopez, Rational matrix approximation with a priori error bounds for non-symmetric matrix Riccati equation with analytic coefficients, IMA J. Numer. Anal. 18 (4) (1998) 545-561.

[7] U. M. Ascher, R. M. Mattheij, R. D. Russell, Numerical Solution of Boundary Value Problems for Ordinary Differential Equations, Prentice-Hall, Englewood Cliffs, NJ, 1988.

[8] I. Babuška, V. Majer, The factorization method for the numerical solution of two point boundary value problems for linear ODE's, SIAM J. Numer. Anal. 24 (1987) 1301-1334.

[9] L. Dieci, M. R. Osborne, R. D. Russell, A Riccati transformation method for solving linear BVPs. I: theoretical aspects, SIAM J. Numer. Anal. 25 (1988) 1055-1073.

[10] L. Dieci, A Riccati transformation method for solving linear BVPs. II: computational aspects, SIAM J. Numer. Anal. 25 (1988) 1074-1092.

[11] A. Graham, Kronecker products and matrix calculus with applications, John Wiley, New York, 1981.

[12] G. H. Golub and C. F. Van Loan, Matrix computations, second ed., The Johns Hopkins University Press, Baltimore, MD, USA, 1989.

[13] F. R. Loscalzo and T. D. Talbot, Spline function approximations for solutions of ordinary differential equations, SIAM J. Numer. Anal. 4 (3) (1967) 433-445.

[14] M. K. Kadalbajoo and K. C. Patidar, Numerical solution of singularly perturbed two-point boundary value problems by spline in tension, Appl. Math. Comput. 131 (2002) 299-320.

[15] E. A. Al-Said and M. A. Noor, Cubic splines method for a system of third-order boundary value problems, Appl. Math. Comput. 142 (2003) 195-204. 
[16] K. R. Raslan, M. A. Ramadan and M. A. Shaalan, Numerical solution of second order matrix differential equations using basis splines, J. Math. Comput. Sci. 6 (6) (2016) 1210-1220.

[17] E. Defez, L. Soler, A. Hervas, and C. Santamaria, Numerical solutions of matrix differential models using cubic matrix splines, Comput. Math. Appl. 50 (2005) 693-699.

[18] E. Defez, L. Soler, A. Hervas, and M. M. Tung, Numerical solutions of matrix differential models using cubic matrix splines II, Mathematical and Computer Modelling 46 (2007) 657-669.

[19] M. M. Tung, E. Defez, and J. Sastre, Numerical solutions of second-order matrix models using cubic-matrix splines, Computers and Mathematics with Applications 56 (2008) 2561-2571.

[20] B. J. McCartin, Theory of exponential splines. Journal of Approximation Theory (66) (1991) 1-23.

[21] R. D. Multire, solution exponential b- splines and singularly perturbed boundary problem. Numer. Algorithms 47 (2008) $191-210$.

[22] O. Ersoy, I. Dag, The exponential cubic b-spline algorithm for Korteweg-de vries equation, Adv. Numer. Anal. (2015) $1-8$.

[23] R. Mohammadi, Exponential b-spline collocation method for numerical solution of the generalized regularized long wave equation, Chin. Phys. B 24 (5) (2015) 050206.

[24] I. Dag, O. Ersoy, The exponential cubic B-spline algorithm for Fisher equation. Chaos, Solitons and Fractals 86 (2016) 101-106.

[25] M. Abbas, A. Abd. Majid, A. I. Md. Ismail, A. Rashid, The application of cubic trigonometric B-spline to the numerical solution of the hyperbolic problems, Appl. Math. Comput. 239 (2014) 74-88.

[26] M. Abbas, A. Abd. Majid, A. I. Md. Ismail, A. Rashid, Numerical method using cubic trigonometric B-Spline technique for non-classical diffusion problem, Abstr. Appl. Anal. 2014 (2014), Article ID 849682, 1-11.

[27] T. Nazir, M. Abbas, A. I. Md. Ismail, A. Abd. Majid, A. Rashid, The numerical solution of advection-diffusion problems using new cubic trigonometric B-splines approach, Applied Mathematical Modelling 40 (2016) 4586-4611.

[28] T. Ak, S. Dhawan, S. B. G. Karakoc, S. K. Bhowmik, K. R. Raslan, Numerical study of Rosenau-KdV equation using finite element method based on collocation approach, Mathematical Modelling and Analysis 22 (3) (2017) 373-388.

[29] T. Ak, S. B. G. Karakoc, H. Triki, Numerical simulation for treatment of dispersive shallow water waves with RosenauKdV equation, The European Physical Journal Plus 131 (10) (2016) 356-370. 